\title{
OGDH is involved in sepsis induced acute lung injury through the MAPK pathway
}

\author{
Yuewei Hao ${ }^{1}$, Zheng Wang ${ }^{2}$, Xinfang Wang ${ }^{3}$, Wenming Zhan ${ }^{4}$, Dianshui $\mathrm{Wu}^{5}$ \\ ${ }^{1}$ Department of Emergency, Shandong Second Provincial General Hospital, Jinan, China; ${ }^{2}$ Prehospital Emergency, Shandong Second Provincial \\ General Hospital, Jinan, China; ${ }^{3}$ Neonatal Intensive Care Unit, Shandong Second Provincial General Hospital, Jinan, China; ${ }^{4}$ Department of \\ Internal Medicine-Cardiovascular, Xishui People's Hospital, Xishui, China; ${ }^{5}$ Department of Clinical Laboratory, Shandong Second Provincial \\ General Hospital, Jinan, China \\ Contributions: (I) Conception and design: Y Hao, D Wu; (II) Administrative support: D Wu; (III) Provision of study materials or patients: Z Wang; (IV) \\ Collection and assembly of data: X Wang; (V) Data analysis and interpretation: W Zhan; (VI) Manuscript writing: All authors; (VII) Final approval of \\ manuscript: All authors. \\ Correspondence to: Dianshui Wu. Department of Clinical Laboratory, Shandong Second Provincial General Hospital, No. 4 Duanxing West Road, \\ Huaiyin District, Jinan 250000, China. Email: 94754206@163.com.
}

Background: Acute lung injury (ALI) induced by sepsis is a common cause of death in clinical practice, and there remains a lack of clinical effective treatment. Cecal ligation and puncture (CLP) is a classic animal model of sepsis, which can induce ALI. Studies have shown that in the lung injury cell model, OGDH (oxoglutarate dehydrogenase) transcription is up-regulated, which is a potential therapeutic target for acute pneumonia. The purpose of this study was to confirm the effects of OGDH on lung injury and inflammation in animal and cell models, and to explore its mechanism.

Methods: By analyzing the GSE16650 gene set, the upregulated OGDH gene was detected in the lung injury cell model. In a sepsis animal model established by CLP and a lung injury cell model, RTPCR, immunohistochemistry, WB, and other techniques were used to verify the upregulation of OGDH expression, which was then was down-regulated with shRNA to confirm its relationship with ALI. Further, ELISA, RT-PCR, and WB were used to detect the effect of OGDH on the expression of pro-inflammatory factors including IL-1 $\beta$, IL-6, IL-18, and TNF- $\alpha$. The downstream pathway of OGDH was predicted using KEGG and GSEA tools and verified by WB and immunofluorescence.

Results: The results showed OGDH was highly expressed in a lung injury cell model and the lung tissue of ALI mice induced by CLP, and downregulation of OGDH alleviated sepsis induced ALI. In animal models and cell models, the expression of OGDH was positively correlated with the expression of pro-inflammatory factors. OGDH may act through the MAPK pathway.

Conclusions: Under the pathological condition of sepsis, OGDH amplifies the inflammatory response through the MAPK pathway, releases pro-inflammatory factors, and induces ALI.

Keywords: Oxoglutarate dehydrogenase; cecal ligation and puncture (CLP); acute lung injury (ALI); proinflammatory factors; MAPK pathway

Submitted May 24, 2021. Accepted for publication Jul 02, 2021.

doi: $10.21037 /$ jtd-21-948

View this article at: https://dx.doi.org/10.21037/jtd-21-948

(c) Journal of Thoracic Disease. All rights reserved. 


\section{Introduction}

The mortality rate of multiple organ dysfunction or failure caused by sepsis is extremely high. The lung is the most vulnerable target organ when sepsis occurs, and about 25$50 \%$ of patients with sepsis will progress to acute lung injury (ALI) or even acute respiratory distress syndrome (ARDS). ALI further aggravates the clinical condition of patients with sepsis, and inflammation is one of the important factors causing organ damage and a poor prognosis in sepsis. White blood cells and platelets are activated, causing the release of pro-inflammatory cytokines, damage to vascular endothelial cells, and potentially leading to organ dysfunction. Despite what is known, the exact mechanism of sepsis causing lung injury is still not fully elucidated, and there remains a lack of obvious effective treatment in clinical practice.

As the most widely used and representative animal model of sepsis, cecal ligation and puncture (CLP) is the gold standard for sepsis model research (1). CLP was improved and popularized by Hubbard et al. (2), and standardized in 2009 (3). Rittirsch et al. used a CLP model to study inflammatory factors in sepsis, and the results showed that the pro-inflammatory factors involved in sepsis immunity mainly included IL-1, IL-2, IL-6, and IL-8, while the antiinflammatory factor IL-10 was also involved (3). In recent years, studies have also shown that IL-17 is a key cytokine in sepsis immunity (4). At the same time of the outbreak of an inflammatory response, CLP modelling showed the same immunosuppression as that seen in clinical patients. Studies have confirmed that lymphocyte apoptosis can induce immunosuppression, and inhibition of lymphocyte apoptosis can significantly improve the survival rate of sepsis (5).

The endotoxin produced by sepsis is absorbed rapidly in the intestinal tract, enters the blood circulation, and distributes to all important organs of the body, leading to multiple organ dysfunction. The lungs are the first and most vulnerable target organs to be affected (6), because their anatomical structure is fragile, pulmonary circulation pressure is low, the blood flow rate in the pulmonary vessels is slow, and they receive a large volume of venous flow, all causing increased exposure time to toxic substances and inflammatory factors. When sepsis occurs, many nucleated white bloods cells "bind" in the lung and release inflammatory factors, oxygen free radicals, proteases, and other substances. The excessive release of inflammatory mediators and oxidative stress are the main factors that promote acute lung loss, and these factors can directly or indirectly lead to lung tissue damage, which is an important cause of death in sepsis patients.

With the development of gene microarray and sequencing technology, comparing the transcriptome differences between pathological and physiological tissues is beneficial when searching for the differentially expressed genes related to ALI. Studies have shown that in an LPS-induced lung injury cell model, the mRNA expression of OGDH (Oxoglutarate Dehydrogenase) is increased relative to normal cells, making it a potential therapeutic target for ALI (7). As an important node in the tricarboxylic acid cycle, the OGDH gene encodes the E1 subunit of the ketoglutarate dehydrogenase complex, which mediates decarboxylation of $\alpha$-ketoglutarate, and catalyzes the complete conversion of 2-oxyglutarate to succinyl coenzyme $\mathrm{A}$ and $\mathrm{CO}_{2}$ (8). This reaction occurs mainly in the mitochondria (9). Studies have shown that OGDH is associated with cell damage after cardiac ischemia perfusion (10), and has previously been reported to be associated with developmental delay, hypotonia, dyspraxia, and metabolic decompensation (11-14). Recently, mutations in OGDH have been reported to cause global growth retardation, lactic acid elevation, ataxia, and seizures (15). However, there is no clear evidence that changes in OGDH expression are associated with various types of inflammation.

Based on the above research background, this study aimed to confirm changes in the expression of OGDH in lung tissue in a model of sepsis induced ALI. Subsequently, OGDH was down-regulated by shRNA to verify that its overexpression was a necessary condition for the occurrence of pneumonia. Further, downstream pathways were analyzed by bioinformatics and simply verified. Through the above studies, we expected to clarify the role of OGDH in a sepsis induced ALI model and confirm whether OGDH could be used as a therapeutic target for this symptom. We present the following article in accordance with the ARRIVE reporting checklist (available at https://dx.doi. org/10.21037/jtd-21-948).

\section{Methods}

\section{Bioinformatics analysis}

The expression profile matrix of external validation cohort data set GSE16650 was directly downloaded from the GEO database (https://www.ncbi.nlm.nih.gov/geo/), and the differentially expressed mRNAs between the LPS induced 
group and the control were analyzed by the $\mathrm{R} /$ Bioconductor package of edgeR with the cut-off value of $|\log 2 \mathrm{FC}|>1$ and FDR $<0.01$. DAVID (david.ncifcrf.gov) was used to perform Kyoto Encyclopedia of Genes and Genomes (KEGG) pathway analysis to identify the function of differentially expressed mRNAs in predicting the underlying biological processes, and the results were visualized using $\mathrm{R}$ language. For GSEA analysis, GSEA tools (http://www.broadinstitute. org/gsea) and the gene expression profile from GSE16650 were used to analyze the potential genes influenced by OGDH. The study was conducted in accordance with the Declaration of Helsinki (as revised in 2013).

\section{CLP-induced sepsis mice model}

All mice were caged in a measured environment $\left(23 \pm 2{ }^{\circ} \mathrm{C}\right.$, 12/12-hour light-dark cycle, $55 \%$ relative humidity) with food and water ad libitum, and a CLP-induced sepsis model was established through CLP surgery as previously described (16). Briefly, mice were fixed after anesthetizing, and a $4 \mathrm{~mm}$ midline incision was made to expose the caecum. The caecum was sutured to a distance of $10 \mathrm{~mm}$ from the tip, and punctured using a needle at a distance of $5 \mathrm{~mm}$ from the ligation. The bowel was then repositioned, and the peritoneum and skin were sutured. Sham-operated mice did not undergo the surgery to the caecum. Our experiments were performed under a project license (No.: XYK20201240) granted by the Animal Ethics Committee of Shandong Second Provincial General Hospital, in compliance with Shandong Second Provincial General Hospital guidelines for the care and use of animals.

\section{Cell cultures and treatments}

The human lung epithelial cell line A549 cell was obtained from the ATCC. Cells were cultured in DMEM medium (cat. no.12110, Solarbio, Inc., China) with 10\% FBS (cat. no. S9020, Solarbio, Inc., China) and 1\% streptomycin/ penicillin (cat. no. P1400, Solarbio, Inc., China). After the cells were incubated for 24 hours, $1 \mu \mathrm{g} / \mathrm{mL}$ LPS (cat. no. L8880, Solarbio, Inc., China) was used to induce the inflammation cell model.

\section{Western blot assay}

Protein expression in lung tissue and cells was measured by Western blot. Briefly, a Protein Extraction kit (cat. no. BC3711, Solarbio, Inc., China) was used to extract the total protein, and a BCA assay (cat. no. PC0020, Solarbio, Inc., China) was used to measure the protein concentration. Proteins from each sample were separated by electrophoresis on 10\% SDS-PAGE gels, then transferred to a polyvinylidene fluoride membrane, blocked with Tris-buffered saline with Tween-20 containing 5\% FBS (cat. no. S9020, Solarbio, Inc., China) at $25^{\circ} \mathrm{C}$ for 1 hour. Proteins were then incubated at $4{ }^{\circ} \mathrm{C}$ overnight with primary antibodies against phosphorylated (p-) MAPK1 and total MAPK1 (1:400 dilution, Cat \# L013V3, Cat \#PA529636, custom ordered from Thermofisher, Inc., USA), total OGDH, GAPDH, IL-1beta, IL-6, IL-18, and TNFalpha (all at 1:400 dilution; cat. nos. ab137773, ab9485, ab216995, ab233706, ab243091, and ab183218, Abcam, Inc., USA). GAPDH was used as an internal reference protein. The membrane was washed three times with TBST and incubated with an appropriate horseradish peroxidase (HRP)-conjugated secondary antibody (1:1,000 dilution; cat. no. A0216; Beyotime, Inc., China) at $25^{\circ} \mathrm{C}$ for 1 hour. The membrane was then washed four times with TBST, and protein expression was detected with ECL reagents (Beyotime, Inc., China) using the chemiluminescent imaging system (Bio-rad, Inc., USA).

\section{$R T-q P C R$}

Total RNA was extracted using Trizol reagent then reverse transcribed into DNA using a RT-PCR Kit (cat. no. K1622, Thermo, Inc., USA), according to the manufacturer's instructions. Real-time PCR was performed with a SYBR Green PCR kit (cat. no. AH0401, SparkJade, Inc., China) on a Real-Time PCR System (Bio-Rad, Inc., USA) with the GAPDH gene used as internal control (17). The $2^{-\Delta \Delta C t}$ method was used to analyze the data (18), and the primers for qPCR were purchased from Sangon Biotech Company (Shanghai, China).

\section{HE staining}

HE staining was performed with a Hematoxylin-Eosin/ HE Staining Kit (cat. no.G1120, Solarbio, Inc., China) according to the manufacturer's instructions. Normal lung tissue was clear, the alveoli were intact and free of congestion and edema, and no inflammatory exudation was present. In contrast, tissue in the lung injury mouse model showed inflammatory cell infiltration. Images were captured by an Olympus IX81 microscope (Olympus, Inc., Japan) in bright-field mode. 


\section{Immunobistochemistry}

OGDH expression was detected following standard immunohistochemical protocol as reported elsewhere (19). Briefly, paraffin-embedded slides $(5 \mu M)$ were baked at $60{ }^{\circ} \mathrm{C}$, then dewaxed by xylene and rehydrated by fractionated ethanol. Antigen was extracted, and incubated with $3 \% \mathrm{H}_{2} \mathrm{O}_{2}$ for $10 \mathrm{~min}$. The slides were then sealed with bovine serum for 1 hour, and anti-OGDH antibody (cat. no. ab137773, Abcam, USA; 1:50 dilution in 1\% bovine serum albumin) were then added and incubated at room temperature for 3 hours. The slides were covered with the secondary antibody and placed in a humid chamber for 1 hour, and DAB was added to reveal the staining intensity. Sections were photographed using an Olympus IX81 microscope (Olympus, Inc., Japan).

\section{Enzyme-linked immunosorbent assay (ELISA)}

Levels of IL-1 $\beta$, IL-6, IL-18, and TNF- $\alpha$ were measured in BALF using ELISA kits (cat. no. SEKM-0002, SEKM0007, SEKM-0019, and SEKM-0034, Solarbio, Inc., China) according to manufacturer's instructions. Optical density values were measured at $450 \mathrm{~mm}$ with a microplate reader (Varioskan LUX, ThermoFisher, USA) in each well.

\section{Immunofluorescence assay}

Immunofluorescence was performed following standard immunofluorescence procedures. Briefly, cells were fixed with 4\% methanol (cat. no. P1110, Solarbio, Inc., China) followed by permeabilization with $0.1 \%$ Triton X-100 (cat. no. T8200, Solarbio, Inc., China) at room temperature. The cells were then blocked with $5 \%$ FBS (cat. no. S9020, Solarbio, Inc., China), then incubated $\left(4{ }^{\circ} \mathrm{C}\right)$ with the primary antibody of p-MAPK1 (Cat \#PA5-97359, Thermofisher, Inc., USA; 1:100) overnight. This procedure was followed by incubation with the fluorescent Alexa Fluor $488^{\circledR}$ ("green”) conjugated secondary anti-rabbit IgG (cat. no. 11008, Invitrogen, USA, 1:1,000) for 1 hour at room temperature. After staining with DAPI and final washes with PBS, images were examined and captured using a fluorescence microscope (OLYMPUS, IX71).

\section{Statistical analysis}

Image analysis was conducted using ImageJ software (Fiji, ImageJ 1.53c). GraphPad Prism 8 was used to perform
Statistical analysis and mapping. One-way analysis of variance followed by a Tukey post-hoc test was used to analyze differences between groups. Data were considered significantly different when $\mathrm{P}<0.05$.

\section{Results}

\section{OGDH was highly expressed in the lung injury cell model and sepsis induced ALI tissue}

To detect genes that play a key role in the inflammatory state of lung cells, we screened the GEO database for differentially expressed genes and chose the dataset GSE16650. Genes differentially expressed in the LPSinduced inflammatory model of A549 cells in the data set relative to control cells were screened out and are shown in Figure 1A,1B. Three genes, OGDH, ATRIP, and $A R I D 2$, were screened, and $O G D H$ was upregulated in the inflammatory cell model, which was then validated in an animal model of CLP-induced ALI. The results showed that compared with the sham operation group, the expression of $O G D H$ mRNA in the lung tissues of mice in the ALI group was up-regulated (Figure 1C), the immunohistochemical staining of OGDH protein in the lung tissues of the CLP group was enhanced (Figure 1D), and the WB results showed that the expression of OGDH protein in the lung tissue of the CLP group was up-regulated (Figure 1E).

\section{Downregulation of OGDH alleviates sepsis induced ALI}

To confirm the causal relationship between OGDH and ALI, we used shRNA to down-regulate the expression of OGDH in CLP mice. As shown in Figure 2A, the expression of OGDH protein in mouse lung tissues was detected by WB after nasal infusion of shRNA, and shOGDH could significantly down-regulate the expression of CLP-induced OGDH protein. HE staining results in Figure $2 B$ show that the lung tissue of mice in the CLP + shNC group has extensive inflammatory cell infiltration in the alveolar cavity and interstitium, exudate in the alveolar cavity, thickened alveolar septa, congestion and bleeding in the alveolar and interstitium, and some alveolar collapse and atelectasis, while the lung tissue structure of the sham group and the CLP + shOGDH group was relatively complete. The alveolar space was clear, the cell wall was smooth, and there was no exudation in the alveolar space and interstitium. The acute lung score was significantly lower than that of CLP + shNC group. Subsequently, we detected other lung injury- 


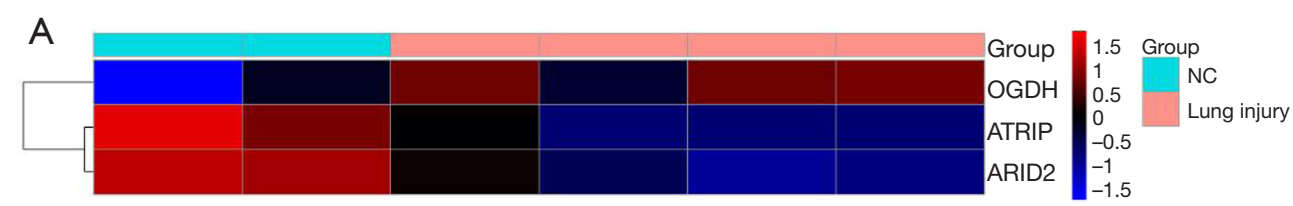

B

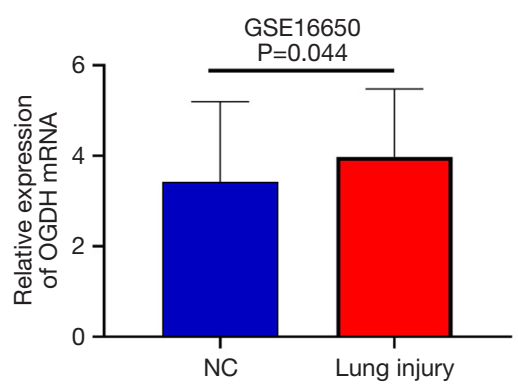

D

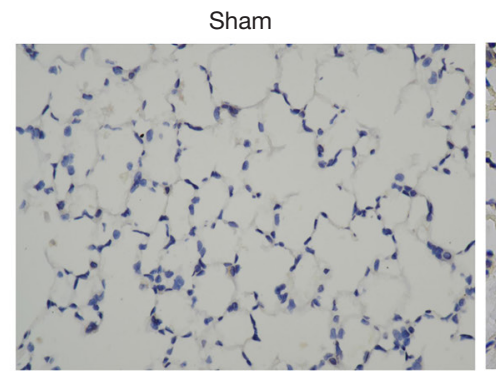

E

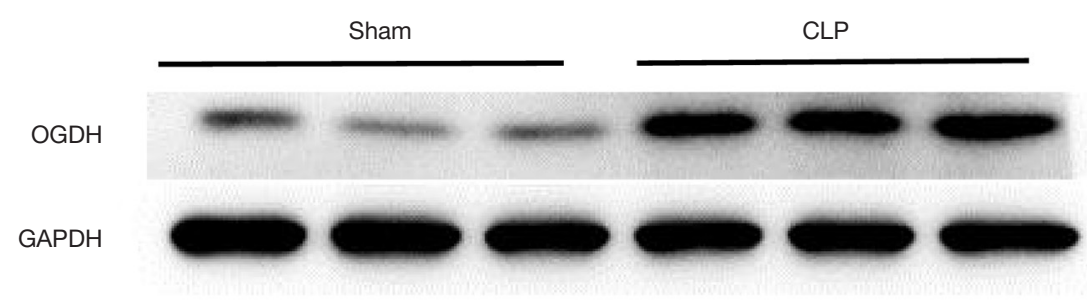

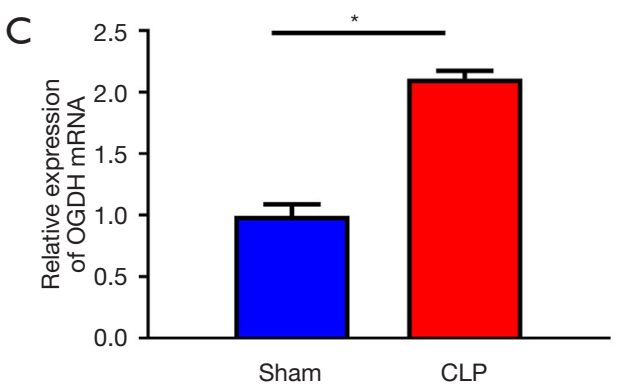

CLP

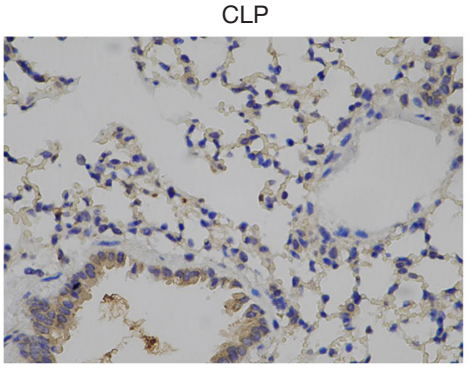

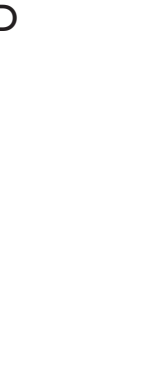


A
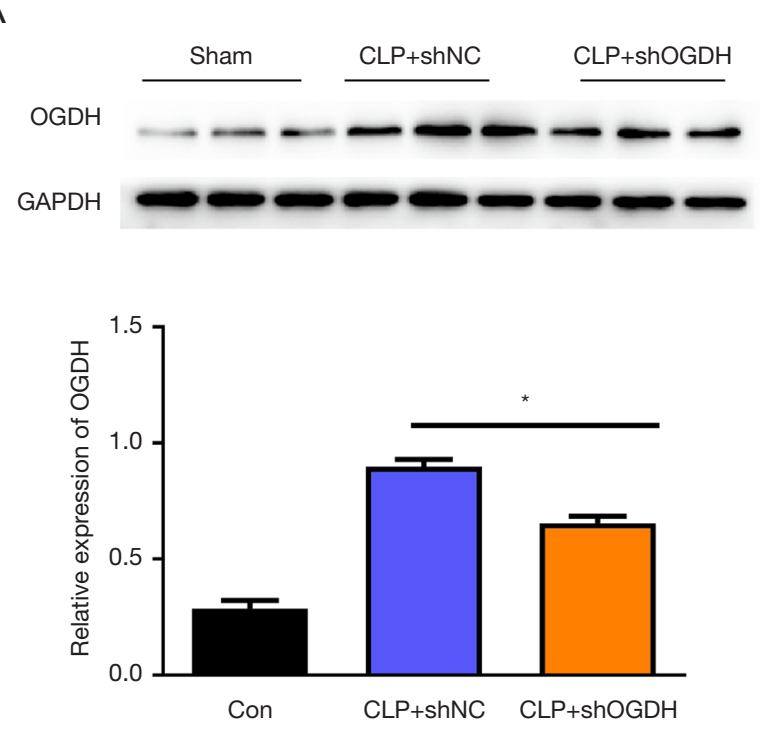

B
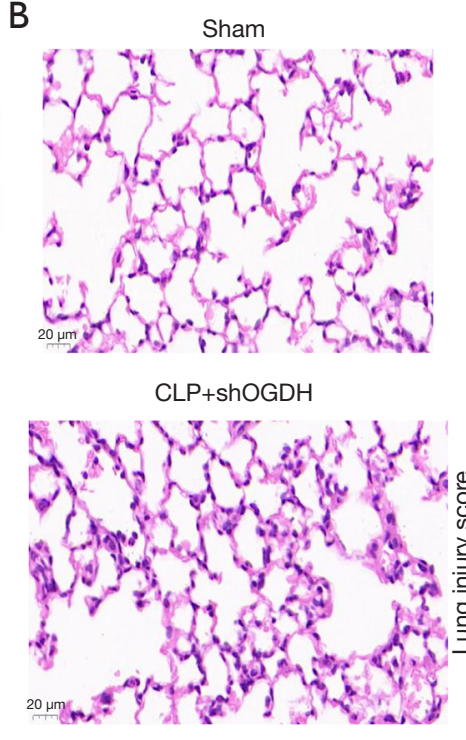
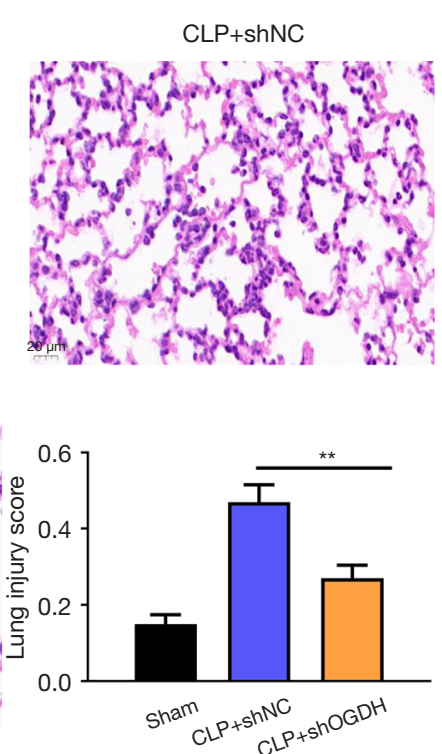

C

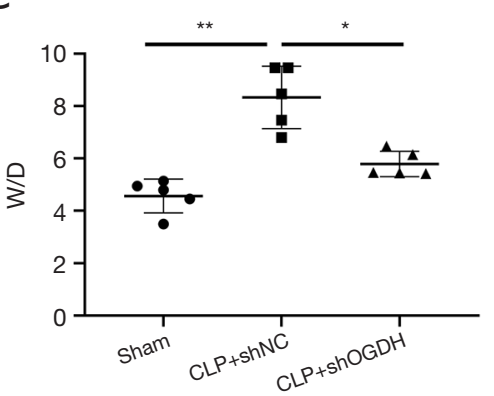

D

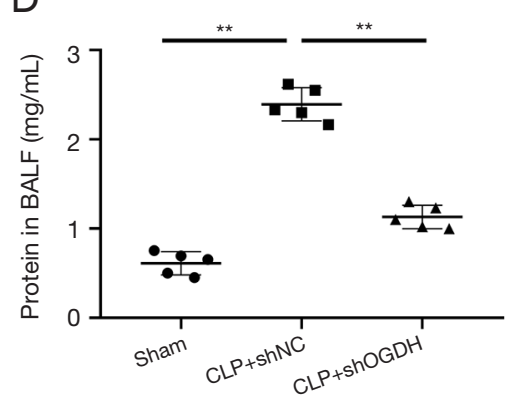

$\mathrm{E}$

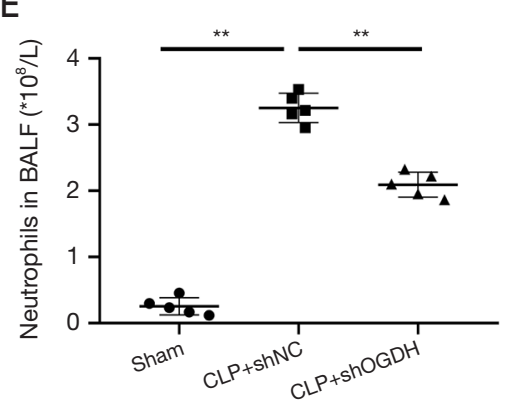

Figure 2 OGDH was highly expressed in lung injury models. (A) shOGDH significantly down-regulated the expression of OGDH in the CLP group; (B) HE staining showed that down-regulation of OGDH significantly reduced lung injury caused by CLP ( $\times 400)$; (C) downregulation of OGDH significantly reduced the increase in the wet-to-dry ratio of lung tissue caused by CLP; (D) downregulation of OGDH significantly reduced the increase of protein content in BALF induced by CLP; (E) downregulation of OGDH significantly reduced the increase of neutrophils in BALF induced by CLP. *, $\mathrm{P}<0.05$; ** $\mathrm{P}<0.01$. OGDH, oxoglutarate dehydrogenase; CLP, cecal ligation and puncture.

with the CLP + shNC group. RT-PCR also showed that shOGDH significantly reversed CLP-induced upregulation of IL-1 $\beta$, IL-6, IL-18, and TNF- $\alpha$ transcriptional levels in lung tissues (Figure 3E-3H). Figure 3I shows that in the WB test, shOGDH significantly reversed the up-regulation of CLP-induced protein expression levels of IL-1 $\beta$, IL-6, IL-18, and TNF- $\alpha$ in lung tissues.

The expression of OGDH and pro-inflammatory cytokine proteins in the LPS-induced pneumonic cell model was consistent with that in the animal model. As shown in Figure $4 A$, LPS treatment induced the upregulation of OGDH expression in A549 cells, and the degree of upregulation was dose-dependent on LPS concentration. Figure $4 B$ shows the expression levels of OGDH and in A549 cells treated with $1 \mu \mathrm{g} / \mathrm{mL}$ LPS increased with the increase in treatment time. The results of the above two cellular inflammation models are consistent with the results of animal models. Figure $4 C-4 G$ shows that the down-regulation of OGDH expression by siOGDH significantly reversed the up-regulation of IL-1 $\beta$, IL-6, IL-18, and TNF- $\alpha$ protein expression in A549 cells induced by LPS. 

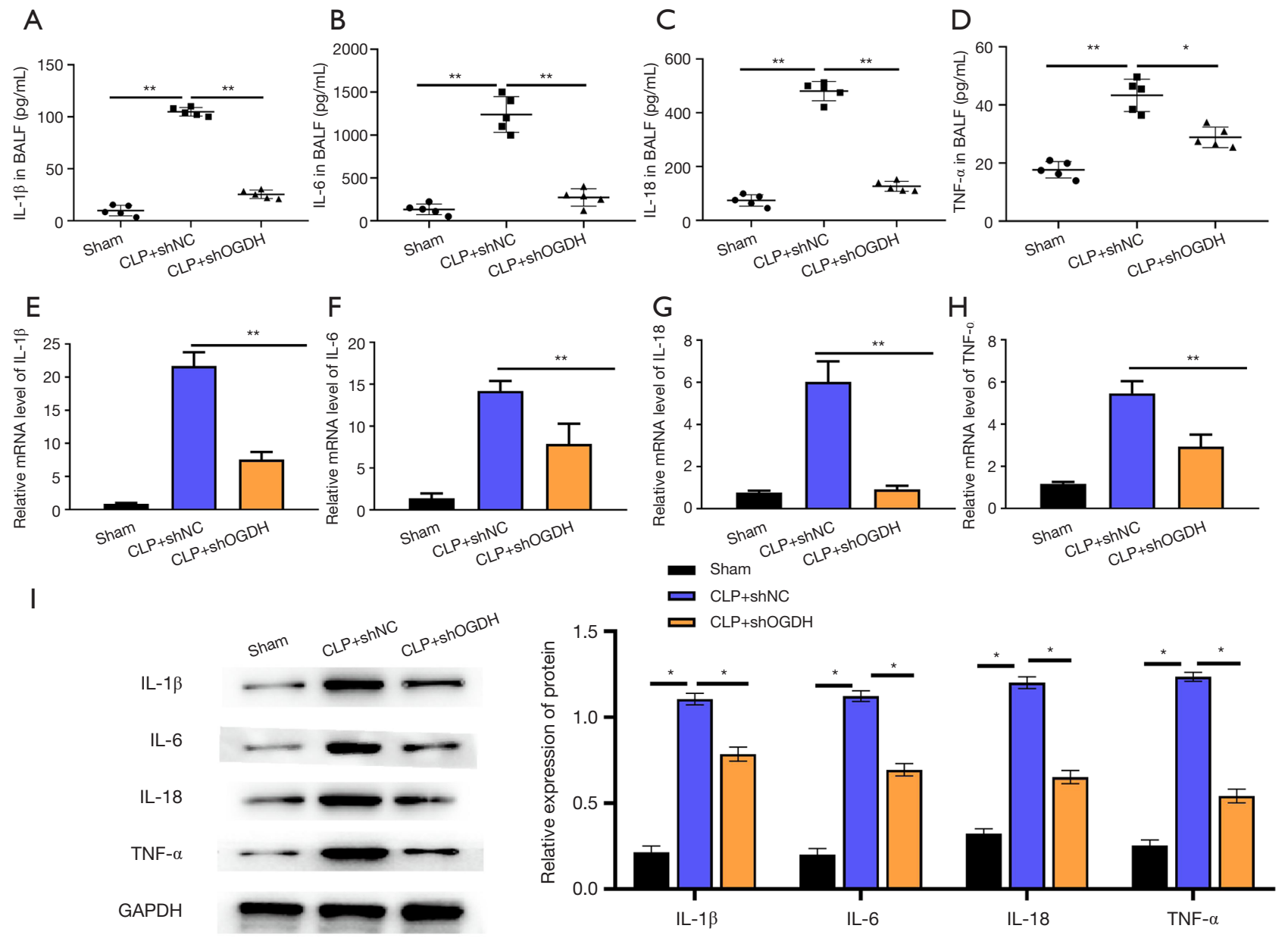

Figure 3 OGDH promotes the expression of pro-inflammatory cytokines in lung cells in animal models. (A) Downregulation of OGDH significantly reduced the increase of IL-1 in BALF induced by CLP; (B) downregulation of OGDH significantly reduced the increase of IL-6 in BALF induced by CLP; (C) downregulation of OGDH significantly reduced the increase of IL-18 in BALF induced by CLP; (D) downregulation of OGDH significantly reduced the increase of TNF-alpha in BALF induced by CLP; (E) Downregulation of OGDH significantly reduced the up-regulation of IL-1 transcription in lung tissue induced by CLP; (F) downregulation of OGDH significantly reduced the up-regulation of IL-6 transcription in lung tissue induced by CLP; (G) downregulation of OGDH significantly reduced the upregulation of IL-18 transcription in lung tissue induced by CLP; $(\mathrm{H})$ downregulation of OGDH significantly reduced CLP-induced TNFalpha transcription upregulation in lung tissue; (I) downregulation of OGDH significantly reduced the up-regulated expression of IL-1, IL-6, IL-18, and TNF-alpha in lung tissue induced by CLP. *, $\mathrm{P}<0.05$; **, $\mathrm{P}<0.01$. OGDH, oxoglutarate dehydrogenase; CLP, cecal ligation and puncture.

\section{OGDH activates the MAPK pathway}

To explore the signaling pathway through which OGDH regulates inflammatory response, we used the KEGG pathway enrichment analysis data set GSE16650, and as shown in Figure 5 A, the differential expressed genes were significantly enriched in the MAPK signaling pathway. GSEA analysis showed that the gene set 'KEGG_MAPK_
SIGNALING_Pathway' was highly expressed in the high expression group of OGDH (Figure 5B), and a CLPinduced ALI model was used for verification. As shown in Figure 5C, the expression of p-MAPK1 in the lung tissue of mice in the CLP + shNC group was up-regulated compared with that in the sham operation group. After the expression of OGDH was down-regulated by shOGDH, that of p-MAPK1 decreased compared with the CLP + 
A

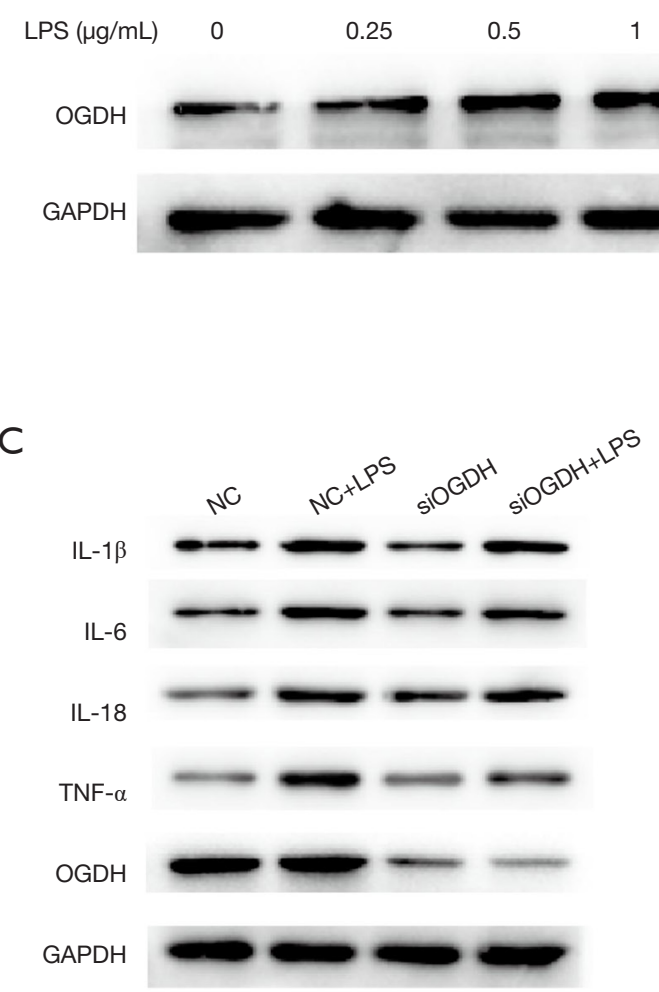

B

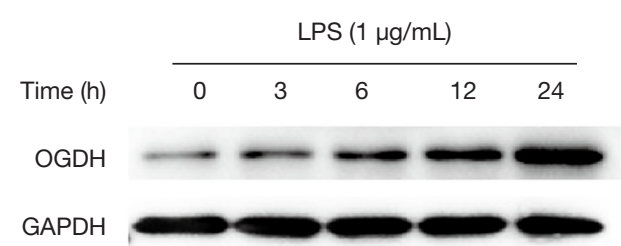

D

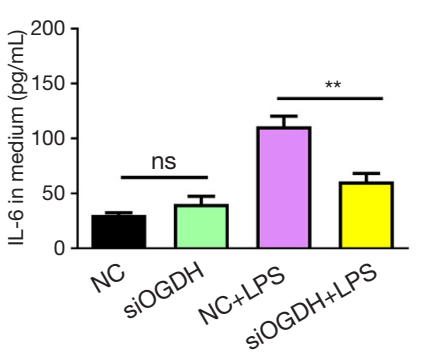

$\mathrm{F}$

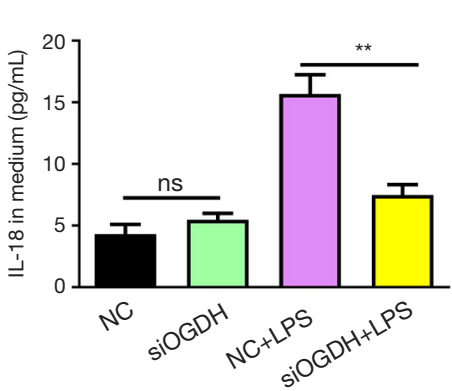

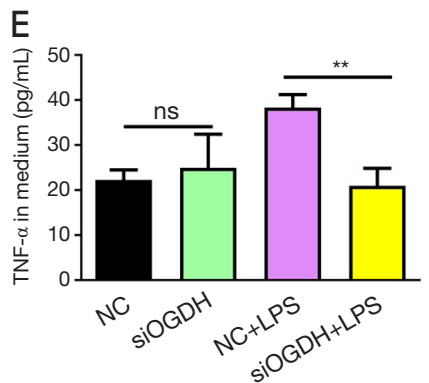

G

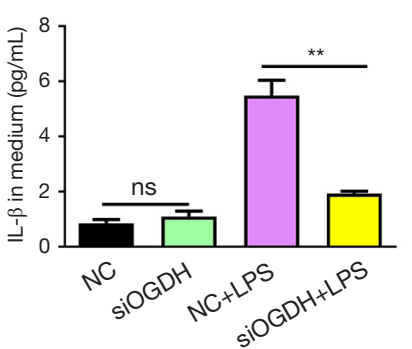

Figure 4 OGDH promotes the expression of pro-inflammatory cytokines in a lung injury cell model. (A) In an LPS-induced pneumonia cell model, the up-regulated expression of OGDH is related to the concentration of LPS treatment. (B) in an LPS-induced pneumonia cell model, the up-regulated expression of OGDH was related to the treatment time of LPS. (C) WB was used to detect the downregulation of OGDH on the expression of pro-inflammatory factors in an LPS-induced pneumonic cell model; (D) downregulation of OGDH significantly reduced the up-regulation of IL-1 transcription induced by LPS; (E) downregulation of OGDH significantly reduced the up-regulation of IL-6 transcription induced by LPS; (F) downregulation of OGDH significantly reduced the up-regulation of IL-18 transcription induced by LPS; $(\mathrm{G})$ downregulation of OGDH significantly reduced the up-regulation of TNF-alpha transcription induced by LPS. **, P<0.01, ns, P $>0.05$. OGDH, oxoglutarate dehydrogenase.

shNC group, and in the LPS-induced pneumonic cell model, the expression of p-MAPK1 was significantly decreased after the down-regulation of OGDH (Figure 5D). Immunofluorescence was used to detect the expression of p-MAPK1 in cells, and the expression of p-MAPK1 was upregulated by LPS treatment, while this could be reversed after siOGDH down-regulation of OGDH (Figure 5E).

\section{Discussion}

Acute lung injury (ALI) remain common complications of sepsis. At this time, a curative treatment strategy for sepsis-induced ALI does not exist. Indeed, the only consensus-approved treatment strategies are early goaldirected therapy for the initial stages of sepsis and activated protein C for severe sepsis (20-22). The only consensusapproved treatment strategy is the use of low tidal volume ventilation after ALI is already established. However, highfidelity animal models of sepsis-induced ALI/ARDS are essential for validating treatment strategies. Takano et al. were confirmed significant protection by pitavastatin on septic ALI thought CLP-induced sepsis mouse model (23). Zhang et al. reported that administration of TSA or SB alleviated sepsis-induced lung injury thought CLP-induced sepsis mouse model (24). In this study, we also established the sepsis animal model by CLP to confirm the effects of OGDH on lung injury and inflammation in animal and cell 


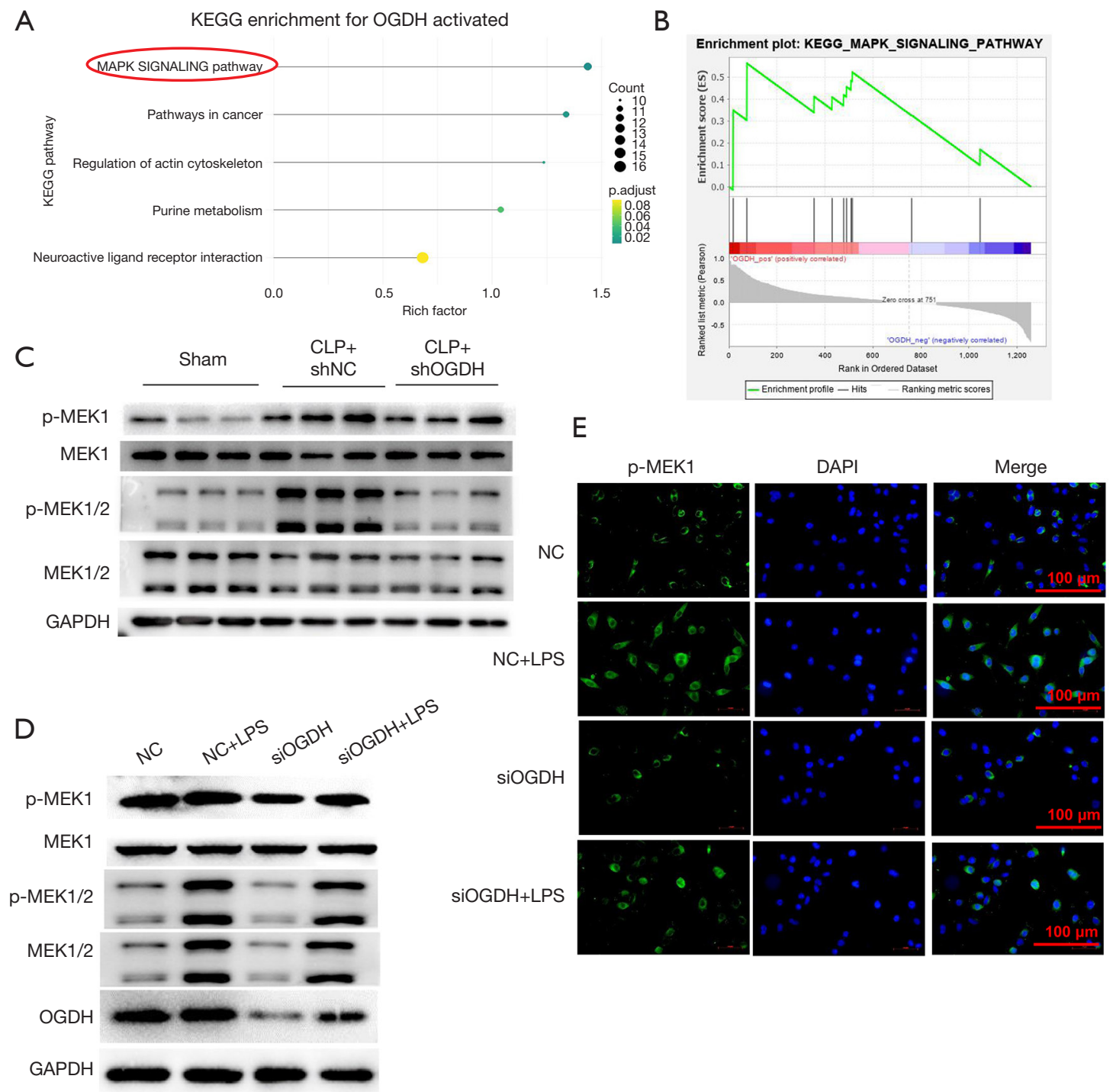

Figure 5 MAPK is the downstream pathway of OGDH. (A) According to the expression data of GSE16650, the KEGG analysis was used to generate the bubble plot; (B) GSEA analysis showed that the gene set 'KEGG_MAPK_SIGNALING_pathway' was highly expressed in the high expression group of OGDH; (C) in the CLP animal model, down-regulation of OGDH reversed the increase of p-MAPK1 expression; (D) in an LPS-induced lung injury cell model, down-regulation of OGDH can down-regulate p-MAPK1 expression; (E) immunofluorescence showed down-regulation of OGDH and down-regulation of p-MAPK1 expression. OGDH, oxoglutarate dehydrogenase.

models, and to explore its mechanism.

In our results, we first screened out the up-regulated $O G D H$ gene in a cellular pneumonia model by analyzing the gene set GSE16650. Subsequently, in an animal model of CLP-induced ALI, OGDH was upregulated in confirmed pneumonia tissues, and shRNA knockdown of the $O G D H$ gene further confirmed that OGDH up- regulation is a necessary condition to cause lung injury. Using ELISA, RT-PCR, WB, and other methods, we identified a positive regulation relationship between OGDH and pro-inflammatory cytokines and detected the upregulation of $\mathrm{OGDH}$ and the corresponding changes in the expression of pro-inflammatory cytokines in a pneumonia cell model. Further, bioinformatics methods were used to 
excavate the gene set GSE16650, and we found that the possible downstream pathway of OGDH was MAPK, which was verified in animal models and pneumonia cell models. In summary, OGDH induces lung injury through upregulation of pro-inflammatory cytokines and is regulated by the MAPK signal transduction pathway.

We used a CLP-induced mouse model of sepsis. Studies have shown that in addition to lung inflammatory damage, CLP-induced model animals also produce organ damage and the rise of pro-inflammatory cytokines in the myocardium, liver, spleen, kidney, and digestive tract (25-31). From the perspective of pathogenesis, the causes that induce sepsis and subsequent organ damage include inflammatory response disorder, immune dysfunction, mitochondrial damage, coagulation dysfunction, neuroendocrine-immune network abnormality, endoplasmic reticulum stress, and autophagy (32-36). The 2-oxoglutarate dehydrogenase complex which catalyzes the overall conversion of 2-oxoglutarate to succinyl-CoA and $\mathrm{CO}_{2}$, is mainly active in the mitochondrion $(8,9)$. As one of the key enzymes in the tricarboxylic acid cycle, the abnormal expression level of OGDH leads to abnormal mitochondrial function, then induces the increase of reactive oxygen species, autophagy, and apoptosis, which may be one of the reasons for subsequent organ damage in sepsis. In another study, OGDH acted as a pro-inflammatory metabolite to increase the production of LPS-induced pro-inflammatory factors through $\mathrm{NF}-\mathrm{\kappa B}$, while IDH2 deficiency resulted in a decrease in OGDH expression, which ultimately reduced the LPS-induced pulmonary inflammatory response (37).

The mitogen-activated protein kinase (MAPK) signaling pathway is one of the most important signal transduction systems in cells, and is involved in cell proliferation, differentiation, apoptosis, and inflammation (38). The phosphorylation of MAPK induces the nuclear translocation of nuclear factor- $\kappa \mathrm{B}$ and induces cell apoptosis (39). At the same time, it induces the expression of adhesion molecules such as ECAM-1 and VCAM-1, and stimulates the production of TNF- $\alpha$, IL- 8 , and monocyte chemotactic protein-1, which initiates and amplifies the inflammatory response of microvascular endothelium during infection (40). Hsieh et al. found that the p38 MAPK signaling pathway exists in most cells and is an important system mediating cellular response, which is involved in cell growth and apoptosis (41). Activated p38 MAPK can regulate the inflammatory response of lung tissue by increasing the recruitment and chemotaxis of neutrophils in vivo and regulating the immune response of macrophages $(42,43)$.
Moreover, activation of p38 MAPK pathway can produce positive feedback with ERK, JNK, and nuclear factorkappaB (NF- $\mathrm{kB}$ ) signaling pathways, and cascade to amplify inflammatory response $(42,44,45)$. By blocking the p38 MAPK signaling pathway, the inflammatory response of lung tissue can be effectively reduced, and alveolar epithelial cells and capillary endothelial cells can be effectively protected (46). Wang et al. reported that Maresin 1 ameliorates sepsisassociated lung injury by inhibiting the activation of the JAK2/STAT3 and MAPK/NF-кB signaling pathways (47). Fang et al. found that modulation of mitogen-activated protein kinase attenuates sepsis-induced acute lung injury in acute respiratory distress syndrome rats (48). Chen et al. reported that Sini decoction ameliorates sepsis-induced acute lung injury via regulating ACE2-Ang (1-7)-Mas axis and inhibiting the MAPK signaling pathway (49).

Based on the above research results and the results of the present study, we speculated that the process of sepsis induced ALI is as follows: Sepsis induces a systemic immune response, immune factors are enriched in lung tissue and affect lung tissue cells, and OGDH up-regulates and activates the MAPK pathway, further amplifying the immune response and damaging cells and tissues. In this study, we identified the relationship between sepsis induced ALI and the expression level of OGDH, the relationship between OGDH and the MAPK pathway, and the relationship between the overexpression of OGDH and the up-regulation of immune factors. However, a series of questions, such as the pathway through which OGDH expression is up-regulated in ALI, the relationship between OGDH expression and cell reactive oxygen species content, and whether the up-regulation of OGDH causes autophagy or apoptosis, have not been clarified. These key questions should be the focus of follow-up studies.

In summary, the possible mechanism of sepsis induced ALI sees OGDH upregulate and activate the MAPK signal transduction pathway and upregulate pro-inflammatory cytokines to induce lung injury.

\section{Acknowledgments}

Funding: None.

\section{Footnote}

Reporting Checklist: The authors have completed the ARRIVE reporting checklist. Available at https://dx.doi. org/10.21037/jtd-21-948 
Data Sharing Statement: Available at https://dx.doi. org/10.21037/jtd-21-948

Conflicts of Interest: All authors have completed the ICMJE uniform disclosure form (available at https://dx.doi. org/10.21037/jtd-21-948). The authors have no conflicts of interest to declare.

Ethical Statement: The authors are accountable for all aspects of the work in ensuring that questions related to the accuracy or integrity of any part of the work are appropriately investigated and resolved. Human research in this study was conducted in accordance with the Declaration of Helsinki (as revised in 2013). Animal experiments were performed under a project license (No.: XYK20201240) granted by the Animal Ethics Committee of Shandong Second Provincial General Hospital, in compliance with Shandong Second Provincial General Hospital guidelines for the care and use of animals.

Open Access Statement: This is an Open Access article distributed in accordance with the Creative Commons Attribution-NonCommercial-NoDerivs 4.0 International License (CC BY-NC-ND 4.0), which permits the noncommercial replication and distribution of the article with the strict proviso that no changes or edits are made and the original work is properly cited (including links to both the formal publication through the relevant DOI and the license). See: https://creativecommons.org/licenses/by-nc-nd/4.0/.

\section{References}

1. Dejager L, Pinheiro I, Dejonckheere E, et al. Cecal ligation and puncture: the gold standard model for polymicrobial sepsis? Trends Microbiol 2011;19:198-208.

2. Hubbard WJ, Choudhry M, Schwacha MG, et al. Cecal ligation and puncture. Shock 2005;24 Suppl 1:52-7.

3. Rittirsch D, Huber-Lang MS, Flierl MA, et al. Immunodesign of experimental sepsis by cecal ligation and puncture. Nat Protoc 2009;4:31-6.

4. Li LL, Dai B, Sun YH, et al. The activation of IL-17 signaling pathway promotes pyroptosis in pneumoniainduced sepsis. Ann Transl Med 2020;8:674.

5. Hotchkiss RS, Monneret G, Payen D. Immunosuppression in sepsis: a novel understanding of the disorder and a new therapeutic approach. Lancet Infect Dis 2013;13:260-8.

6. Zimmerman JJ, Akhtar SR, Caldwell E, et al. Incidence and outcomes of pediatric acute lung injury. Pediatrics
2009;124:87-95.

7. Hu P, Wang X, Haitsma JJ, et al. Microarray meta-analysis identifies acute lung injury biomarkers in donor lungs that predict development of primary graft failure in recipients. PLoS One 2012;7:e45506.

8. Armstrong CT, Anderson JL, Denton RM. Studies on the regulation of the human E1 subunit of the 2-oxoglutarate dehydrogenase complex, including the identification of a novel calcium-binding site. Biochem J 2014;459:369-81.

9. Wang Y, Guo YR, Liu K, et al. KAT2A coupled with the $\alpha-\mathrm{KGDH}$ complex acts as a histone $\mathrm{H} 3$ succinyltransferase. Nature 2017;552:273-7.

10. Pan $Y$, Wang $Y$, Shi W, et al. Mitochondrial proteomics alterations in rat hearts following ischemia/reperfusion and diazoxide post-conditioning. Mol Med Rep 2021;23:161.

11. Bonnefont JP, Chretien D, Rustin P, et al. Alphaketoglutarate dehydrogenase deficiency presenting as congenital lactic acidosis. J Pediatr 1992;121:255-8.

12. Dunckelmann RJ, Ebinger F, Schulze A, et al. 2-ketoglutarate dehydrogenase deficiency with intermittent 2-ketoglutaric aciduria. Neuropediatrics 2000;31:35-8.

13. Guffon N, Lopez-Mediavilla C, Dumoulin R, et al. 2-Ketoglutarate dehydrogenase deficiency, a rare cause of primary hyperlactataemia: report of a new case. J Inherit Metab Dis 1993;16:821-30.

14. Kohlschütter A, Behbehani A, Langenbeck $U$, et al. A familial progressive neurodegenerative disease with 2-oxoglutaric aciduria. Eur J Pediatr 1982;138:32-7.

15. Yap ZY, Strucinska K, Matsuzaki S, et al. A biallelic pathogenic variant in the OGDH gene results in a neurological disorder with features of a mitochondrial disease. J Inherit Metab Dis 2021;44:388-400.

16. Ruiz S, Vardon-Bounes F, Merlet-Dupuy V, et al. Sepsis modeling in mice: ligation length is a major severity factor in cecal ligation and puncture. Intensive Care Med Exp 2016;4:22.

17. Okazaki K, Sakamoto K, Kikuchi R, et al. Mapping and characterization of FLC homologs and QTL analysis of flowering time in Brassica oleracea. Theor Appl Genet 2007;114:595-608.

18. Livak KJ, Schmittgen TD. Analysis of relative gene expression data using real-time quantitative PCR and the 2(-Delta Delta C(T)) Method. Methods 2001;25:402-8.

19. Pagliari M, Munari F, Toffoletto $M$, et al. Helicobacter pylori Affects the Antigen Presentation Activity of Macrophages Modulating the Expression of the Immune Receptor CD300E through miR-4270. Front Immunol 2017;8:1288. 
20. Russell JA. Management of sepsis. N Engl J Med 2006;355:1699-713.

21. Rhee C, Klompas M. Sepsis trends: increasing incidence and decreasing mortality, or changing denominator? J Thorac Dis 2020;12:S89-S100.

22. Rivers E, Nguyen B, Havstad S, et al. Early goal-directed therapy in the treatment of severe sepsis and septic shock. N Engl J Med 2001;345:1368-77.

23. Takano K, Yamamoto S, Tomita K, et al. Successful treatment of acute lung injury with pitavastatin in septic mice: potential role of glucocorticoid receptor expression in alveolar macrophages. J Pharmacol Exp Ther 2011;336:381-90.

24. Zhang L, Jin S, Wang C, et al. Histone deacetylase inhibitors attenuate acute lung injury during cecal ligation and puncture-induced polymicrobial sepsis. World J Surg 2010;34:1676-83.

25. Zhang $W, \mathrm{Xu} X, \mathrm{Kao} \mathrm{R}$, et al. Cardiac fibroblasts contribute to myocardial dysfunction in mice with sepsis: the role of NLRP3 inflammasome activation. PLoS One 2014;9:e107639.

26. Protzer U, Maini MK, Knolle PA. Living in the liver: hepatic infections. Nat Rev Immunol 2012;12:201-13.

27. Heymann F, Tacke F. Immunology in the liver--from homeostasis to disease. Nat Rev Gastroenterol Hepatol 2016;13:88-110.

28. Babic ZM, Zunic FZ, Pantic JM, et al. IL-33 receptor (ST2) deficiency downregulates myeloid precursors, inflammatory $\mathrm{NK}$ and dendritic cells in early phase of sepsis. J Biomed Sci 2018;25:56.

29. Zeng X, McMahon GM, Brunelli SM, et al. Incidence, outcomes, and comparisons across definitions of AKI in hospitalized individuals. Clin J Am Soc Nephrol 2014;9:12-20.

30. Zhang H, Wei L, Zhang Z, et al. Protective effect of periplaneta americana extract on intestinal mucosal barrier function in patients with sepsis. J Tradit Chin Med 2013;33:70-3.

31. Wang X, Chen GY, Yang SS, et al. Effects of high thoracic epidural anesthesia on ischemic cardiomyopathy cardiac function and autonomic neural function. Genet Mol Res 2014;13:6813-9.

32. Takeuchi O, Akira S. Pattern recognition receptors and inflammation. Cell 2010;140:805-20.

33. Rocha M, Herance R, Rovira S, et al. Mitochondrial dysfunction and antioxidant therapy in sepsis. Infect Disord Drug Targets 2012;12:161-78.

34. Ma Y, Zhou Y, Wu F, et al. The Bidirectional Interactions
Between Inflammation and Coagulation in Fracture Hematoma. Tissue Eng Part B Rev 2019;25:46-54.

35. Borovikova LV, Ivanova S, Zhang $M$, et al. Vagus nerve stimulation attenuates the systemic inflammatory response to endotoxin. Nature 2000;405:458-62.

36. Jiao G, Hao L, Wang M, et al. Upregulation of endoplasmic reticulum stress is associated with diaphragm contractile dysfunction in a rat model of sepsis. Mol Med Rep 2017;15:366-74.

37. Park JH, Ku HJ, Lee JH, et al. Disruption of IDH2 attenuates lipopolysaccharide-induced inflammation and lung injury in an $\alpha$-ketoglutarate-dependent manner. Biochem Biophys Res Commun 2018;503:798-802.

38. Cao J, Gong Y, Cai B, et al. Modulation of human bronchial epithelial cells by pneumococcal choline binding protein A. Hum Immunol 2011;72:37-46.

39. Walley KR. Sepsis-induced myocardial dysfunction. Curr Opin Crit Care 2018;24:292-9.

40. Fiuza C, Bustin M, Talwar S, et al. Inflammationpromoting activity of HMGB1 on human microvascular endothelial cells. Blood 2003;101:2652-60.

41. Hsieh CC, Papaconstantinou J. Thioredoxin-ASK1 complex levels regulate ROS-mediated p38 MAPK pathway activity in livers of aged and long-lived Snell dwarf mice. FASEB J 2006;20:259-68.

42. Li X, Zhou X, Ye Y, et al. Lyn regulates inflammatory responses in Klebsiella pneumoniae infection via the $\mathrm{p} 38 /$ NF- $\kappa$ B pathway. Eur J Immunol 2014;44:763-73.

43. Ittner A, Block H, Reichel CA, et al. Regulation of PTEN activity by p38ס-PKD1 signaling in neutrophils confers inflammatory responses in the lung. J Exp Med 2012;209:2229-46.

44. Kang H, Bang TS, Lee JW, et al. Protective effect of the methanol extract from Cryptotaenia japonica Hassk. against lipopolysaccharide-induced inflammation in vitro and in vivo. BMC Complement Altern Med 2012;12:199.

45. Monteggia LM, Luikart B, Barrot M, et al. Brain-derived neurotrophic factor conditional knockouts show gender differences in depression-related behaviors. Biol Psychiatry 2007;61:187-97.

46. Sim YS, Kim SY, Kim EJ, et al. Impaired Expression of MAPK Is Associated with the Downregulation of TNF- $\alpha$, IL-6, and IL-10 in Mycobacterium abscessus Lung Disease. Tuberc Respir Dis (Seoul) 2012;72:275-83.

47. Wang F, Wang M, Wang J, et al. Maresin 1 ameliorates sepsis-associated lung injury by inhibiting the activation of the JAK2/STAT3 and MAPK/ NF- $\mathrm{B}$ signaling pathways. 
Microb Pathog 2020;148:104468.

48. Fang W, Cai SX, Wang CL, et al. Modulation of mitogen-activated protein kinase attenuates sepsis-induced acute lung injury in acute respiratory distress syndrome rats. Mol Med Rep 2017;16:9652-8.

Cite this article as: Hao Y, Wang Z, Wang X, Zhan W, Wu D. $\mathrm{OGDH}$ is involved in sepsis induced acute lung injury through the MAPK pathway. J Thorac Dis 2021;13(8):5042-5054. doi: $10.21037 /$ jtd-21-948
49. Chen Q, Liu J, Wang W, et al. Sini decoction ameliorates sepsis-induced acute lung injury via regulating ACE2Ang (1-7)-Mas axis and inhibiting the MAPK signaling pathway. Biomed Pharmacother 2019;115:108971. 